\title{
Nuclear density-functional theory and fission of super-heavy elements
}

\author{
P.-G. Reinhard ${ }^{1}$ \\ Institut für Theoretische Physik, Universität Erlangen, Staudtstrasse 7, D-91058 Erlangen, Germany
}

August 10, 2021/ Received: date / Revised version: date

\begin{abstract}
We review the prediction of fission properties of super-heavy elements (SHE) by self-consistent mean-field models thereby concentrating on the widely used Skyrme-Hartree-Fock (SHF) approach. We explain briefly the theoretical tools: the SHF model, the calibration of model parameters together with statistical analysis of uncertainties and correlations, and the involved computation of fission lifetimes. We present an overview of fission stability in comparison to other decay channels over the whole landscape of SHE reaching deep into the $r$-process domain. The main emphasis lies on a detailed discussion of the various ingredients determining eventually the fission properties. The main result is that fission is an involved process which explores many different influences with almost equal share, basic bulk properties (also known as liquid-drop model parameters), pairing strengths, and shell effects.
\end{abstract}

PACS. 2 1.10.Tg, 21.60.Jz, 25.85.Ca

\section{Introduction}

Since mid last century, it became possible to produce new elements not naturally appearing on earth. This triggered intense research on transactinides 1 proceeding with time to ever heavier nuclei, coined super-heavy elements (SHE) $[2,3$. The search is still going on and more new elements are added every year to the list of SHE, see e.g. 4, 5, 6,, 7 8. Knowledge of exotic nuclei beyond the valley of stability is crucial in understanding astro-physical reactions and amongst them properties of SHE are important in order to determine the upper end of the nucleosynthesis flow 9 , 10,11. No surprise then that SHE constitute an extremely active field of experimental and theoretical research. The Frankfurt theory group under W. Greiner joined these efforts from the theoretical side rather early [12,13 and continued since. The theoretical methods were much developing over the years. Today's standard, in nuclear structure generally and also for SHE, are self-consistent meanfield models in terms of nuclear density functional theory (DFT) which came up in the 1970ies and have meanwhile reached high descriptive power for nuclear structure and dynamics, for reviews see e.g. [11, 14, 15, 16, 17.

The key question in the study of SHE is their stability, particularly against spontaneous fission. First estimates of stability were obtained from analyzing shell structure. The ultimate test, of course, is a direct analysis of fission barriers and lifetimes. The basic mechanism of nuclear fission as a many-body tunneling process proceeding along steadily changing nuclear shapes has been understood in terms of phenomenological shell models since long [2,18. However, self-consistent calculations of fission lifetimes are ex- tremely demanding and thus have come up only recently, see e.g. 19, 20, 21 for calculations with approximate collective masses or 22, 23 for fully self-consistent calculations and for a recent review 24. It is the aim of this contribution to discuss predictions from self-consistent models on fission barriers and lifetimes with a quick glance at the competing reactions $\alpha$ - and $\beta$-decay and neutron emission. Within the self-consistent models, we concentrate in particular on the Skyrme-Hartree-Fock approach. We discuss in detail its predictive power using methods of statistical analysis related to the phenomenological calibration of the model.

The paper is outlined as follows: In section 2 , we summarize the Skyrme-Hartree-Fock (SHF) model, the computation of fission lifetimes, and explain statistical analysis for estimating extrapolation uncertainties and parameter correlations. In section 3, we present and discuss the results starting from a quantitative overview of various decay channels followed by an extensive discussion of error estimates and correlations.

\section{Formal framework}

\subsection{The Skyrme mean-field model}

We consider here nuclear DFT using the SHF energydensity functional $\mathcal{E}_{\mathrm{Sk}}(\rho, \tau, \mathcal{J}, \mathbf{j}, \sigma)$, which is expressed in terms of a few local densities and currents obtained as sums over single-particle wave functions: density $\rho$, kinetic density $\tau$, spin-orbit density $\mathcal{J}$, current $\mathbf{j}$, spin density $\sigma$, 
and pairing density $\xi$. This reads, e.g., for the density

$$
\rho_{q}(\mathbf{r})=\sum_{\alpha \in q} f_{\alpha} v_{\alpha}^{2}\left|\varphi_{\alpha}(\mathbf{r})\right|^{2}
$$

where $q \in\{p, n\}$ labels proton or neutron density, $\varphi_{\alpha}$ are s.p. wavefunctions, $v_{\alpha}^{2}$ the corresponding pairing weights, and $f_{\alpha}$ pairing phase-space factors (see below). It is convenient to formulate the functional in terms of isoscalar density $\rho_{0}=\rho_{n}+\rho_{p}$ and isovector density $\rho_{1}=\rho_{n}-\rho_{p}$. The same holds for the other densities and currents.

Using these densities, the total energy is composed as

$$
\begin{aligned}
E=E_{\mathrm{kin}}+ & \int d^{3} r \mathcal{E}_{\mathrm{Sk}}+E_{\mathrm{Coul}}+E_{\mathrm{pair}}-E_{\mathrm{corr}}, \\
E_{\mathrm{kin}}= & \int d^{3} r\left(\frac{\hbar^{2}}{2 m_{p}} \tau_{p}+\frac{\hbar^{2}}{2 m_{n}} \tau_{n}\right) \\
\mathcal{E}_{\mathrm{Sk}}= & C_{T}^{\rho} \rho_{T}^{2}+C_{T}^{\rho, \alpha} \rho_{T}^{2} \rho_{0}^{\alpha}+C_{T}^{\Delta \rho} \rho_{T} \Delta \rho_{T} \\
& +C_{T}^{\tau} \rho_{T} \tau_{T}+C_{T}^{\nabla J} \rho_{T} \nabla \cdot \mathbf{J}_{T}+C_{T}^{J} \mathbf{J}_{T}^{2}, \\
E_{\mathrm{Coul}}= & \frac{e^{2}}{2} \int d^{3} r d^{3} r^{\prime} \frac{\rho_{p}(\mathbf{r}) \rho_{p}\left(\mathbf{r}^{\prime}\right)}{\left|\mathbf{r}-\mathbf{r}^{\prime}\right|} \\
& +\frac{3 e^{2}}{4}\left(\frac{3}{\pi}\right)^{1 / 3} \int d^{3} r\left[\rho_{p}(\mathbf{r})\right]^{4 / 3}
\end{aligned}
$$

where $e^{2}=1.44 \mathrm{MeV}$ fm. The $\mathcal{E}_{\mathrm{Sk}}$ here shows, for simplicity, only the part containing time-even densities, because this defines already the basic model parameters $C_{T}^{(\text {typ })}$ and only this part counts in stationary calculations. The tensor spin-orbit term $\propto C_{T}^{J}$ is, in fact, more involved [25. But its details have little impact on basic bulk properties. Thus it is usually ignored at all with setting $C_{T}^{J}=0$. For a detailed discussion of the functional, its parameters, time-odd terms and other options see [17].

The correlation energy $E_{\text {corr }}$ may contain several contributions. The least to do is the correction for the spurious center-of-mass energy $E_{\mathrm{cm}}=\frac{1}{2 m A}\left\langle\hat{\mathbf{P}}_{\mathrm{cm}}^{2}\right\rangle$ where $m A$ is the total mass and $\hat{\mathbf{P}}_{\mathrm{cm}}=\sum_{n=1}^{A} \hat{\mathbf{p}}_{n}$ the total momentum. The $\hat{\mathbf{P}}_{\mathrm{cm}}^{2}$ is, in fact, a two-body operator. Some Skyrme parametrizations simplify that by using only its diagonal term of, for details see [15, 17. Deformed nuclei require also a correction for rotational projection and soft surface vibrations [26,27]. These will be discussed in connection with the fission path in section 2.4 .

The pairing functional reads

$$
\mathcal{E}_{\text {pair }}=\frac{1}{4} \sum_{q \in\{p, n\}} V_{\text {pair,q }} \int d^{3} r \xi_{q}^{2}\left[1-\frac{\rho}{\rho_{0, \text { pair }}}\right]
$$

Letting $\rho_{0 \text {,pair }} \rightarrow \infty$ suppresses the density-dependent part and recovers what is called volume pairing. Surface pairing is obtained by setting $\rho_{0 \text {,pair }}=0.16 \mathrm{fm}^{-3}$. Most

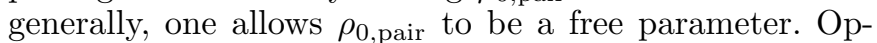
timizing the pairing functional this way, one obtains as optimum a mix of volume and surface pairing [28. The pairing functional $3 \mathrm{a}$ ) is complemented by a cutoff in s.p. space [29]. We use a soft cutoff factor

$$
f_{\alpha}=\left[1+\exp \left(\left(\varepsilon_{\alpha}-\left(\epsilon_{F}+\epsilon_{\text {cut }}\right)\right) / \Delta \epsilon\right)\right]^{-1},
$$

where $\Delta \epsilon=\epsilon_{\text {cut }} / 10, \epsilon_{\alpha}$ is the single particle energy of the state $\alpha$ and $\epsilon_{F}$ is the chemical potential. The original recipe used a fixed cutoff energy $\epsilon_{\text {cut }}=5-10 \mathrm{MeV}$ 30,31. Here, we determine $\epsilon_{\text {cut }}$ such the the active s.p. space has a fixed size $\propto N^{2 / 3}$, yielding a cutoff which depends on the actual nucleon number $N_{q}$ [32]

$$
\epsilon_{\text {cut }} \longleftrightarrow \sum_{\alpha} f_{\alpha}=N_{q}+\eta_{\text {cut }} N_{q}^{2 / 3}
$$

We use $\eta_{\text {cut }}=1.65$ in the following calculations.

The mean-field equations are derived variationally from the given energy functional. Pairing is performed at the level of the BCS approximation.

The SHF functional sets only a framework. The model parameters $C_{T}^{(\text {typ })}$ remain yet to be determined. In the early days of nuclear DFT, the quality of nuclear ab-initio calculations was poor. Thus the parameters of the functional had been adjusted empirically. The most systematic way to do this are least-squares $\left(\chi^{2}\right)$ fits 33 used for nuclear DFT first in 34 and being meanwhile a standard technique for calibration of self-consistent mean-field models. One finds in the literature a great variety of functional development using $\chi^{2}$-fits to empirical data. For example, some concentrate on spherical nuclei with negligible correlation effects [28, others are particularly concerned with deformed nuclei $[35,36,37$, still others try to adjust also spectra of s.p. energies [38,39]. Although differences occur in details due to different choice of fit data, it is reassuring that all these fits provide similar and highquality results for the reproduction of global nuclear properties. Nuclear many-body theory has made in the meantime enormous progress, see e.g. 40,41,42. Nonetheless, the precision of these ab-initio models does not yet suffice for a high-quality description of nuclear properties. Thus it is still state of the art to adjust the DFT parameters empirically. We will address that briefly in section 2.3 .

The parameters of the SHF functional 2 ce are initially the $C_{T}^{(\text {typ })}$. Some of them can be associated with an immediate meaning. For example, the $C_{T}^{\Delta \rho}$ characterize surface tension and $C_{T}^{\nabla J}$ the spin-orbit strength. The volume parameters, however, are less transparent. It is advantageous to express them through the nuclear matter parameters (NMP), i.e. the basic properties of symmetric matter: the ground state properties equilibrium binding energy $E / A_{\mathrm{eq}}$ and equilibrium density $\rho_{\text {eq }}$, the static response properties incompressibility $K$, symmetry energy $J$ and density dependence of symmetry energy $L$, and the dynamic response properties (isoscalar) effective mass $\mathrm{m}^{*} / m$, and Thomas-Reiche-Kuhn sum-rule enhancement $\kappa_{\text {TRK }}$ which characterizes isovector effective mass, for a detailed definition see e.g. [17]. There is a one-to-one mapping between the SHF volume parameters and NMP

$$
C_{0,1}^{\rho}, C_{0,1}^{\rho, \alpha}, \alpha,\left.C_{0,1}^{\tau} \Leftrightarrow \frac{E}{A}\right|_{\mathrm{eq}}, \rho_{\mathrm{eq}}, K, J, L, \frac{m^{*}}{m}, \kappa_{\mathrm{TRK}}
$$




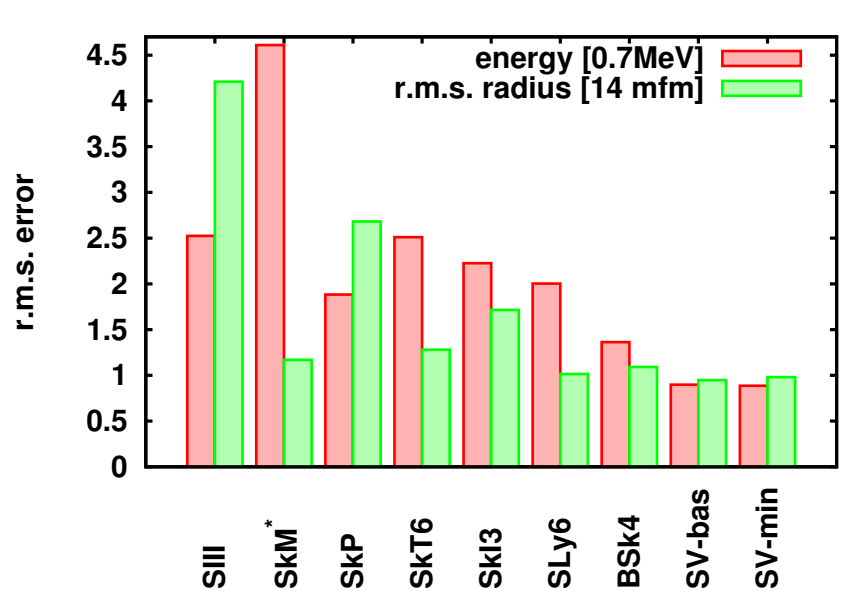

Fig. 1. Quality of a couple of Skyrme parametrizations in terms of r.m.s. error on binding energy and charge r.m.s. radius. The parametrizations are sorted along time of publication: SIII 43, SkM* 44, SkP 45], SkT6 46], SkI3 47], SLy6 48, BSk4 [9], SV-bas and SV-min [28. The average errors are computed over the large pool of fit data from 28 which contains semi-magic nuclei over a wide range of sizes and isotopes.

such that both ways of handling the model parameters are equivalent. We will use later on the form in terms of NMP.

\subsection{Variety of SHF parametrizations}

Empirical adjustment leaves some freedom in the choice of reference data and relative weights of them. Thus there exists in the literature a variety of parametrizations. Figure 1 compares a selection of typical and widely used parametrizations as they have developed in the course of time. Means of comparison is the quality of binding energy and charge r.m.s. radii in terms of the r.m.s. deviation to experimental data for the selection of semi-magic nuclei of 28. The figure shows how parametrizations have improved over the decades. This is due to gathering more experience with systematic fits over the years and, more important, to the appearance of new data on exotic nuclei which help enormously to pin down the properties of a functional. A comment is in order about the two earliest parametrizations. The first one, SIII, concentrated on energies with the consequence that these are already quite well reproduce while the radii are a bit off. The next move, SkM*, worked on a correct reproduction of excitation properties and fission barriers. This involves a large improvement on r.m.s. radii with sacrifices at the side of energies. Nonetheless, $\mathrm{SkM}^{*}$ served for a long period almost as standard because it provides a well equilibrated reproduction of a large set of observables. From then on, improvements did proceed steadily and slowly and the process is still going on. In the meantime came up parametrizations which come down to an average error on energies of $0.5 \mathrm{MeV}$ and on r.m.s. radii on $0.012 \mathrm{fm} 50$. However, this often requires corrective terms not included in the present SHF model and the gain is subtle. Thus we

\begin{tabular}{|l|cr|rrr|}
\hline & $K$ & $m^{*} / m$ & \multicolumn{1}{c|}{$L$} & $\kappa_{\mathrm{TRK}}$ \\
\hline Gogny D1 & 229 & 0.67 & 31 & 18 & \\
\hline SkI3 & 258 & 0.58 & 35 & 212 & 0.25 \\
SLy6 & 230 & 0.69 & 32 & 100 & 0.25 \\
SkT6 & 236 & 1.00 & 30 & 64 & 0.00 \\
SkM$^{*}$ & 217 & 0.79 & 30 & 95 & 0.53 \\
SkP & 201 & 1.00 & 30 & 41 & 0.35 \\
BSk4 & 237 & 0.92 & 28 & 27 & 0.18 \\
SV-bas & 234 & 0.90 & 30 & 68 & 0.40 \\
SV-min & 222 & 0.95 & 31 & 93 & 0.08 \\
UNEDF2 & 240 & 0.93 & 29 & 40 & 0.25 \\
\hline NL-Z2 & 175 & 0.58 & 39 & 126 & 0.72 \\
DD-PC & 185 & 0.57 & 35 & 82 & 0.75 \\
DD-ME & 250 & 0.56 & 32 & 6 & 0.79 \\
\hline
\end{tabular}

Table 1. Nuclear matter parameters (NMP) for the for the various parametrizations used in this paper. The $K, J$, and $L$ are given in $\mathrm{MeV} ; m^{*} / m$ and $\kappa_{\mathrm{TRK}}$ is dimensionless. In case of RMF (last three entries), $m^{*} / m$ and $\kappa_{\text {TRK }}$ stand for the values at momentum $k=0$.

confine the present study to the above list of parametrizations. After all, we emphasize that most parametrizations deliver a comparable and good description of ground-state data. They differ, however, in other features. Table 1 exemplifies this for NMP. Similar variances of results are also seen for giant resonances, isotopic trends, and particularly fission barriers which will be discussed later. In addition to the above listed parametrizations, we will employ in section 3.3 a set with a systematic variation of model parameters.

\subsection{Calibration and statistical analysis}

The principles of $\chi^{2}$-fits are simple. The model under consideration, here SHF, produces for given model parameters $\mathbf{p}$ a great manifold of expectation values of observables $A=A(\mathbf{p})$. We select a subgroup of fit observables $\hat{\mathcal{O}}_{f}$ for which we have reliable experimental data and from which we assume that they can be described reliably well by DFT. The quality of the model is quantified by

$$
\chi^{2}(\mathbf{p})=\sum_{f} \frac{\left(\mathcal{O}_{f}(\mathbf{p})-\mathcal{O}_{f}^{(\exp )}\right)^{2}}{\Delta \mathcal{O}_{f}}
$$

where $f$ runs over the pool of fit data, $\hat{\mathcal{O}}_{f}^{(\exp )}$ stands for the corresponding experimental values, and $\Delta \mathcal{O}_{f}$ is an adopted error which regulates the relative weight of different observables. It accounts mainly for the expected theoretical reliability for this observable and to lesser extend also to the experimental uncertainty. There is an automatic feedback built in: The adopted errors $\Delta \mathcal{O}_{f}$ are chosen correctly if the final $\chi^{2}$ is of order of the number of data points (for a detailed discussion see [33,51]). The best fit is obviously given by that parametrization $\mathbf{p}_{0}$ which minimizes $\chi^{2}$. What thus remains is to find the absolute minimum of the multi-dimensional function $\chi^{2}(\mathbf{p})$. 


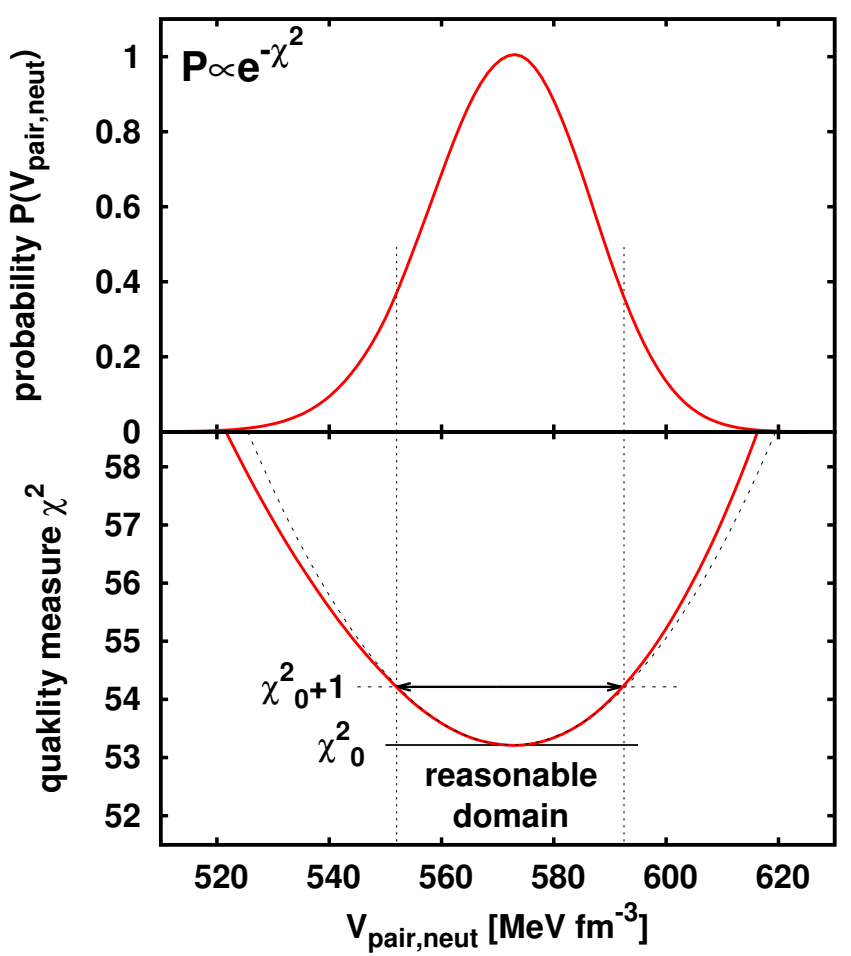

Fig. 2. Illustration of the statistical interpretation of $\chi^{2}$. Lower panel: $\chi^{2}$ for the case of the parametrization SV-min 28. as function of $V_{\text {pair,n }}$; the fine dashed line indicates the quadratic approximation around the minimum. Upper panel: corresponding probability $P$ of the parametrization.

This is a non-trivial task for which, however, a great deal of know-how is around.

There is more in the $\chi^{2}$-scheme than just determining the minimum $\chi_{0}^{2}=\chi^{2}\left(\mathbf{p}_{0}\right)$. The vicinity of the minimum carries also worthwhile information. Parameters which lie near the minimum can still provide a reasonable good description. How good depends, again, an the actual $\chi^{2}$ landscape. This is illustrated in figure 2 whose lower panel shows a cut through the landscape along the pairing parameter $V_{\text {pair,n }}$. It is obvious that $\chi^{2}$ remains acceptably low in the immediate neighborhood of the minimum. This is quantified in terms of the corresponding "probability of a parametrization" $P(\mathbf{p})$ whose cut along $V_{\text {pair,n }}$ is shown in the upper panel. The $P(\mathbf{p})$ leads to the statistical interpretation of $\chi^{2}$. The maximum probability $P$ is found, of course, for minimal $\chi^{2}$ and the curvature of $\chi^{2}$ around the best fit $\mathbf{p}_{0}$ determines the speed of growth. The range of reasonable parameters is defined as the area of $\mathbf{p}$ for which $\chi^{2} \leq \chi^{2}\left(\mathbf{p}_{0}\right)+133$. The $\chi^{2}(\mathbf{p})$ near the minimum is close to a parabolic function (compare full and dashed line in the lower panel of figure 2). Thus the range of reasonable parameters covers an ellipsoid in $\mathbf{p}$-space. The probability distribution $P(\mathbf{p})$ allows to compute statistical averages of any observable $A=A(\mathbf{p})$. For the average, we have usually $\bar{A}=\int d \mathbf{p} P(\mathbf{p}) A(\mathbf{p}) \approx A\left(\mathbf{p}_{0}\right)$. The uncertainty
$\Delta A=\sqrt{\Delta^{2} A}$ of an observable is obtained simply from

$$
\Delta^{2} A=\overline{(A-\bar{A})^{2}}=\int d \mathbf{p} P(A-\bar{A})^{2} .
$$

It quantifies the extrapolation uncertainty propagated from the uncertainties of the model parameters. Still more information can be gained from mixed uncertainties $\overline{\Delta A \Delta B}$ computed in similar fashion. They allow to deduce the amount of statistical correlation, also called alignment between two observables. It is defined as

$$
r_{A B}=\frac{\overline{\Delta A \Delta B}}{\sqrt{\overline{\Delta^{2} A}} \sqrt{\overline{\overline{\Delta^{2} B}}}} .
$$

A value $r_{A B}= \pm 1$ means that the two observables are fully (anti-)correlated, i.e. knowledge of $B$ does not add any new information to knowledge of $A$ (within the given model). In contrast, a value $r_{A B}=0$ means that $A$ and $B$ are uncorrelated, thus fully independent within the model. Often, one is not interested on the sign of $r_{A B}$ and considers the coefficient of determination (CoD) $r_{A B}^{2}$ [52]. This is what we will present in the results later on.

Correlation analysis is compact and instructive, but often too compact. A visual impression of correlations can be visualized by trend analysis. An example will be given in section 3.3

\subsection{Constrained mean field and the collective path}

Fission is an extension of collective quadrupole motion to finally two fragments. It proceeds through a succession of quadrupole deformed mean fields, called the collective path. We generate the path by imprinting a dedicated deformation using quadrupole-constrained mean-field equations

$$
\left(\hat{h}-\epsilon_{\mathrm{F}} \hat{N}-\lambda \hat{Q}_{20}\right)\left|\Phi_{\alpha_{20}}\right\rangle=\mathcal{E}\left|\Phi_{\alpha_{20}}\right\rangle,
$$

where $\hat{h}$ is the SHF mean field Hamiltonian (depending on the local densities), $\epsilon_{\mathrm{F}}$ is the Fermi energy, and $\lambda$ the Lagrange parameter for the quadrupole constraint. The $\hat{Q}_{20}$ is the quadrupole operator and $\alpha_{20}$ its dimensionless expectation value, i.e.

$$
\begin{aligned}
\hat{Q}_{20} & =r^{2} Y_{20} g_{\text {cut }}(\mathbf{r}), \\
\alpha_{20} & =\frac{4 \pi}{5} \frac{\left\langle\Phi_{\alpha_{20}}\left|r^{2} Y_{20}\right| \Phi_{\alpha_{20}}\right\rangle}{A r^{2}},
\end{aligned}
$$

with $A$ the total particle number and $r$ the r.m.s. radius. Note that we consider here only axially symmetric quadrupole deformations, i.e. only the 0 component of the more general $\alpha_{2 m}$. The quadrupole operator is modified by a damping function $g_{\text {cut }}$ which cuts the quadratic growth at large distances to avoid artifacts from deep wells at the bounds of the numerical box [53. The equations are solved with an extra iterative loop to maintain a wanted value of $\alpha_{20}$ 54. This is done for a dense set of deformations 


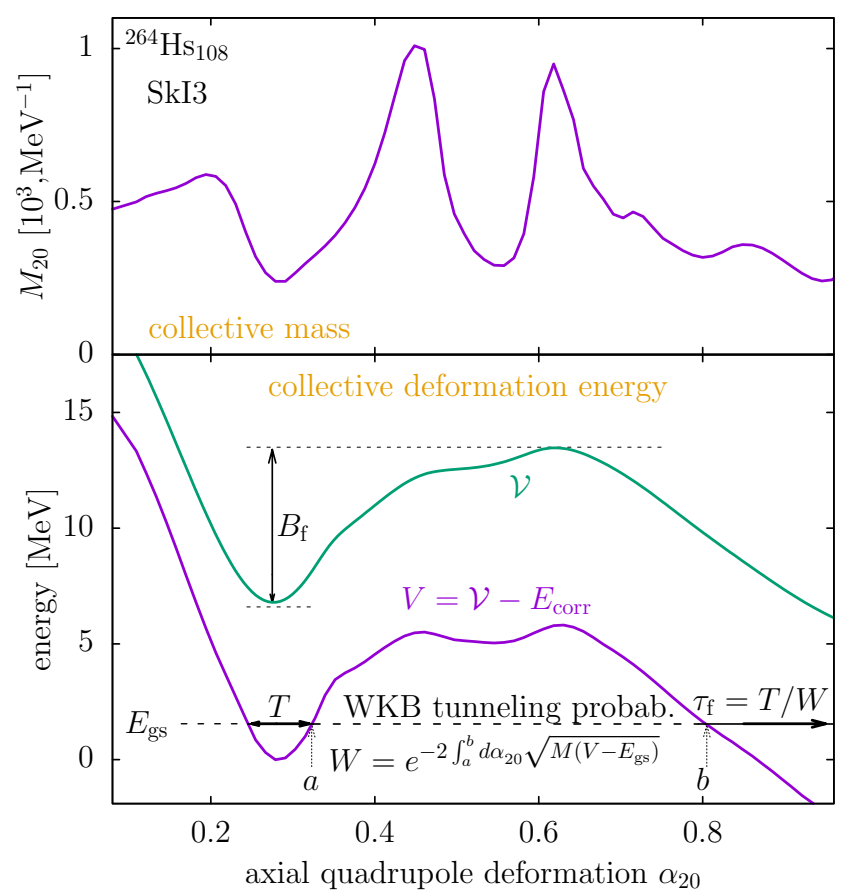

Fig. 3. Potential energy surface (lower panel) and inverse collective mass (upper panel) along the fission path for ${ }^{264} \mathrm{Hs}$ computed with the SHF parametrization SkI3 47. For the potential energy, one distinguishes the raw energy $\mathcal{V}$ and the fission potential $V$ including quantum correction, see eq. (9). The ground-state energy $E_{\mathrm{gs}}$ is indicated by a horizontal line, dashed in the classically forbidden (tunneling) regime and full in the classically allowed regime. The limits of the tunneling regime are indicated by $a$ and $b$ and $W$ is the tunneling probability. The $T$ stands for the oscillation time of the ground state within the binding pocket and $\tau_{\mathrm{f}}$ is the fission lifetime.

$\alpha_{20}$ which yields the collective path as a series of meanfield states $\left\{\left|\Phi_{\alpha_{20}}\right\rangle\right\}$ along which the collective motion can evolve.

Having the path, we can compute a raw collective potential $\mathcal{V}$ as as the SHF energy along the path states, i.e. $\mathcal{V}\left(\alpha_{20}\right)=E\left[\Phi_{\alpha_{20}}\right]$. The self-consistent evaluation of the collective mass $M\left(\alpha_{20}\right)$ for motion along $\alpha_{20}$, also called ATDHF mass, is rather involved as it requires to compute the dynamical response to changing shape, for details see [27,55]. In similar fashion, one computes also the momentum of inertia $\Theta$ for rotation orthogonal to the nuclear axis. With these collective masses and widths, $\mu_{\text {quad }}\left(\alpha_{20}\right)=\left\langle\Phi_{\alpha_{20}}\left|\hat{P}_{20}^{2}\right| \Phi_{\alpha_{20}}\right\rangle$ and $\mu_{\text {rot }}\left(\alpha_{20}\right)=\left\langle\Phi_{\alpha_{20}}\right| \hat{J}_{x}^{2}+$ $\hat{J}_{y}^{2}\left|\Phi_{\alpha_{20}}\right\rangle$, we compute the quantum correction to the potential yielding finally the true collective potential as

$$
V\left(\alpha_{20}\right)=\mathcal{V}\left(\alpha_{20}\right)-\frac{\mu_{\text {quad }}}{2 M}-\frac{1}{4 \lambda} \partial_{\alpha_{20}}^{2} \mathcal{V}-\frac{\mu_{\text {rot }}}{2 \Theta} .
$$

With $V\left(\alpha_{20}\right), M\left(\alpha_{20}\right)$, and $\Theta\left(\alpha_{20}\right)$, we dispose of all ingredients for the collective Hamiltonian which determines quadrupole vibrations and fission [27, 22, 55].

The above review of fission calculations is very sketchy. The treatment of pairing, in particular, covers more sub- tleties. One point is that the BCS approximation to full HFB weakens if a considerable amount of continuum states becomes occupied. This may play a role for the weakly bound nuclei at the edges of the large scans of isotopes in the following. But the observed trends still remain relevant and BCS is valid for the well bound SHE which are discussed in quantitative detail. A more important point concerns controlling of particle. A projection on exact particle number would be ideal. This, however, is inhibited for typical nuclear density functionals 56. BCS provides at least conservation of particle number in the average by virtue of the particle-number constraint. Thus all states along the path represent the same average proton and neutron number. To maintain this crucial feature for the coherent superposition of states in the collective ground state and along fission, we carry forth the particle-number constraint into the collective Schrödinger equation. This somewhat involved procedure is explained in great detail in 22, 23] and reviewed briefly in figure 3. The basic structure of a fission potential is already set by the raw potential $\mathcal{V}$, namely a binding pocket at some small deformation followed by a growth towards a maximum (barrier) then turning to a steady decrease due to unhindered Coulomb repulsion. An immediate measure of stability is the fission barrier which we take here for simplicity as $B_{\mathrm{f}}=\mathcal{V}_{\max }-\mathcal{V}_{\min }$, the difference between maximum and ground-state minimum in the raw potential. The quantum corrections change the potential curve at a quantitative level, typically reducing the barrier by about 1-2 MeV which, however, has sizable consequences for fission lifetimes 26,55,57. The collective mass $M$ has a much fluctuating structure produced by level crossings near the Fermi energy while the momentum of inertia $\Theta$ (not shown) is a smooth function steadily increasing with deformation 58 . Fission lifetime is computed as tunneling dynamics along the collective quadrupole momentum. First, one computes the ground state in the pocket by solving the collective Schrödinger equation in the three dimensions set by deformation $\alpha_{20}$ and two rotation angles (about $x$ and $y$ axis). This defines at fully quantum mechanical level the ground state energy $E_{\mathrm{gs}}$. The repetition rate $T$ for the oscillations in the pocket are computed semiclassically by $T=\hbar \int_{a}^{b} d \alpha_{20} \sqrt{M /\left(V-E_{\mathrm{gs}}\right)}$. The tunneling probability $W$ is calculated in similar fashion, see figure 3 . Both together yield finally the fission lifetime $\tau_{\mathrm{f}}=T / W$. It is to be noted that fission dynamics is computed within three-dimensional quadrupole dynamics related to axially symmetric configurations while full quadrupole dynamics would explore a five-dimensional space covering also triaxial shapes. The limitation is justified because the fission process follows a rather narrow fission valley. We checked that a five-dimensional calculation of the ground state does not make much of a difference in the regime SHE 22,23 .

After all, we see that fission lifetime $\tau_{\mathrm{f}}$ is a highly complex observable composed from many different ingredients. Pocket and barrier are generated by an interplay of Coulomb pressure and shell effects [18. The collective mass gathers influences of all level crossings along the 
path. The collective ground state energy determines the repetition rate $T$ and entry point $a$ as well as exit point $b$ of the tunneling region whose width $b-a$ has large impact on the tunneling probability $W$. Considering all these highly sensitive influences, it is more than surprising that theory can deliver at all a decent value for $\tau_{\mathrm{f}}$. However, we should be prepared to see large differences in predictions with tiny variations of the models parametrization.

\section{Results and discussion}

\subsection{Systematics of barriers and lifetimes over the landscape of SHE}

We start the presentation with, so to say, the final result and we use for that the SHF parametrization SVmin which promises to provide reliable estimates of fission properties, as we will work out later. Figure 4 shows the systematics of quantities characterizing the stability of SHE: fission barriers, fission lifetimes, $\alpha$-decay lifetimes, and neutron separation energies. The computation of fission barrier and lifetimes was explained in section 2.4. The $\alpha$ - and $\beta$-decay lifetimes $\tau_{\alpha}$ are computed using the Viola Seaborg-formula [59]. The $\beta$-decay lifetimes $\tau_{\beta}$ were computed in perturbation theory evaluating explicitly the $\beta$-transition matrix elements to the final odd-odd nucleus, for details see 23 . The neutron separation energies are simply the energy differences between the given nucleus and the neighboring odd nucleus obtained by removing one neutron. For the thus necessary computation of oddodd and odd-even nuclei see 60 .

The nuclear landscape covered in figures 4 reaches deep into the region of neutron instability (see the panels neutron separation energies and $\beta$ halflifes). Note that the neutron rich region cannot be reached in a laboratory on earth but may play a role at the upper end of the astro-physical $r$ process. Let us look first at fission barriers and lifetimes. At first glance, they show, of course, the same trends. Large fission stability is seen at regions of near shell closures $(\mathrm{Z} / \mathrm{N} \approx 108 / 150, \mathrm{~N} \approx 184)$ and toward extremely large neutron numbers, deep in the $r$ process region. Between the islands of stability extend swamps of extremely short fission lifetimes. These quick fluctuations of fission lifetimes over the landscape are due to shell effects. Besides these shell fluctuations, there is an interesting trend with proton number. With increasing proton number $Z$, fission lifetimes become comparatively shorter. The point is that barrier height $B_{\mathrm{f}}$ is not everything. The width of the barrier has also large impact on the tunneling probability $W$ and the widths shrink toward increasing $Z$. Even though, we still encounter considerable fission stability at the upper island $\mathrm{Z} / \mathrm{N} \approx 120 / 180$.

The $\alpha$-decay lifetimes show smooth trends throughout and in the average they have similar order of magnitude as fission lifetimes. This yields an interesting interplay with fission lifetimes. Near the islands of fission stability, $\alpha$ decay prevails. But the corresponding $\alpha$-chains are interrupted as soon as they cross a swamp of fission instability.
This is a feature which complicates experimental assessment of SHE. The same interplay is seen at the extreme neutron rich side. Both lifetimes, fission and $\alpha$-decay, become very large there. This may give a chance for accumulation of SHE in stellar matter. We have to keep in mind, however, that barriers and $Q_{\alpha}$ may change in the presence of neutron gas 61 .

\subsection{Barriers for various parametrizations}

A simple measure for fission stability is the fission barrier. Figure 5 shows the variety of $B_{\mathrm{f}}$ for ${ }^{266} \mathrm{Hs}$ as predicted by different mean-field models, actually the same selection for which we had shown the average quality in figure 1. The span of $B_{\mathrm{f}}$ values is large. But recall that the selection covers different generations of parametrizations and that the calibration of models has much improved over the years. The figure indicates two crucial NMP. Parametrizations with $m^{*} / m \approx 0.9$ and $J \approx 30 \mathrm{MeV}$ deliver best $B_{f}$. Lower $m^{*} / m$ enhance shell effects, thus delivering larger shellcorrection energies at minimum and consequently larger barriers. There occurs a curious coincidence for the RMF parametrization NL-Z2. The effective mass is extremely low, but $\mathrm{J}$ is extremely high. Both unusual NMP together happen to produce a correct $B_{\mathrm{f}}$.

The error bars on the $B_{\mathrm{f}}$ for SV-bas and SV-min in figure 5 show extrapolation uncertainties obtained by statistical analysis (section 2.3). Both are smaller than the span of results from the selection of parametrizations. This is due to the fact that the large pool of data in the recent fits produces better confined model parameters and, in turn, smaller uncertainties in predicted observables. The large difference in uncertainties between SV-bas and SVmin is explained by the different fit data. While SV-min is fitted to ground-state data of finite nuclei only, SVbas fixes additionally the four NMP $K, m^{*} / m, J$, and $\kappa_{\text {TRK }}$ (which is equivalent to fit giant resonances and polarizability in ${ }^{208} \mathrm{~Pb}[28$ ). Putting more information into SV-bas naturally reduces uncertainties. Interesting is the remarkable reduction as compared to SV-min. This indicates that these four NMP together have a large impact on fission barriers. This will be disentangled in more detail in the following subsections.

\subsection{Impact of model parameters - trend analysis}

A simple and instructive way to explore the impact of model parameters is trend analysis. To this end, one defines a base parametrization, chooses one particular model parameter, and varies it systematically around the base point. Here we take as base point SV-bas 28 which was fitted where four NMP, namely $K, m / m, J$, and $\kappa_{\mathrm{TRK}}$, had been kept fixed during the $\chi^{2}$ fit. Then one takes one of these frozen NMP, sets it to a slightly different value, and refits all other model parameters. This is done for a couple of different values thus delivering a set of parametrizations with systematically varied DFT parameter. Then one can draw other observables as functions of 


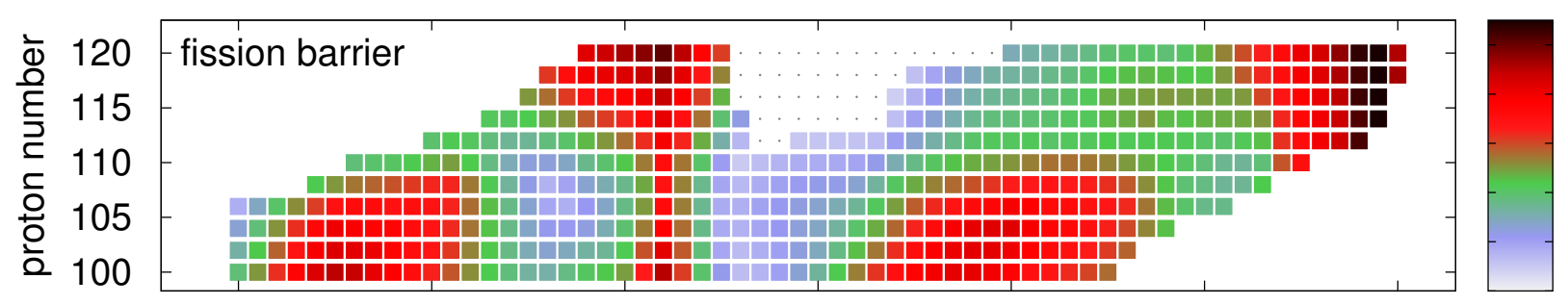

10

$8>$

$6 \sum^{\infty}$

4

2

0
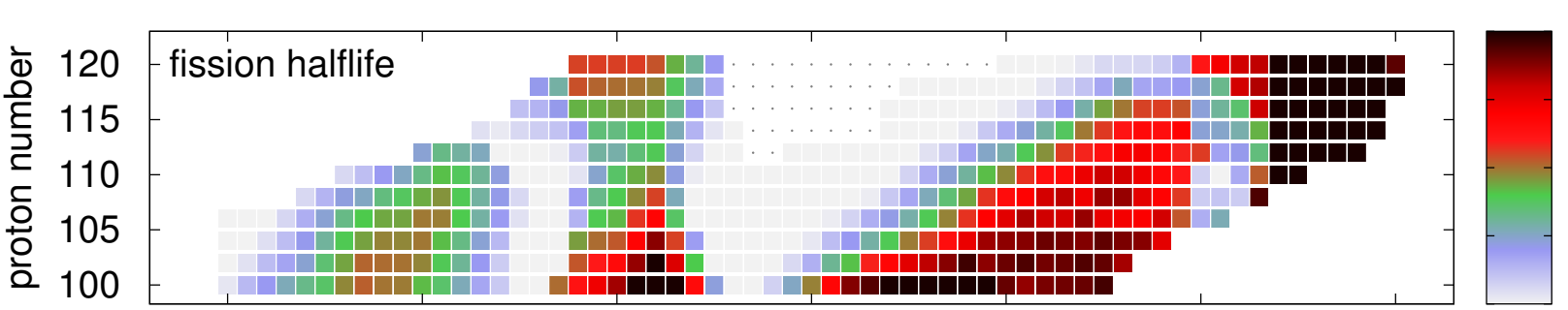

$10^{12}$

$10^{6}$

$1 \frac{\bar{\omega}}{\stackrel{\omega}{\omega}}$

$10^{-6}$

$10^{-12}$

$10^{12}$
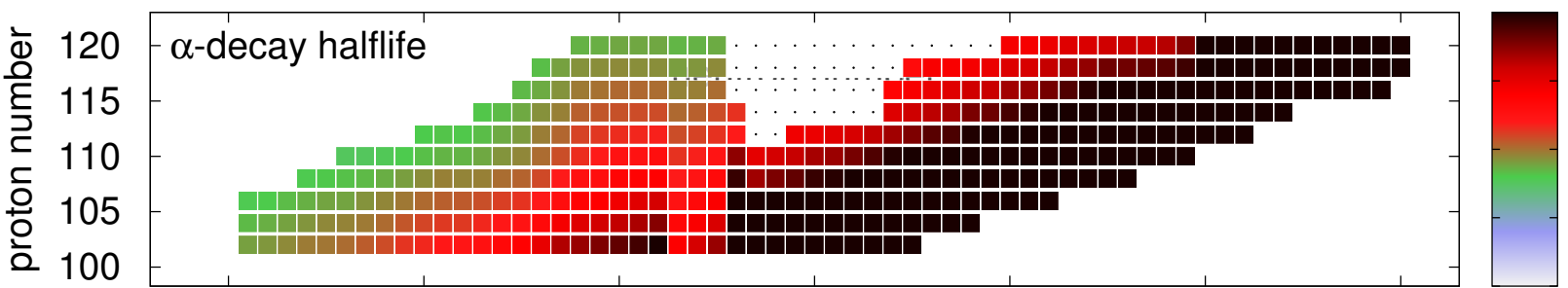

$10^{6}$

$1 \frac{\bar{s}}{8}$

$10^{-6}$
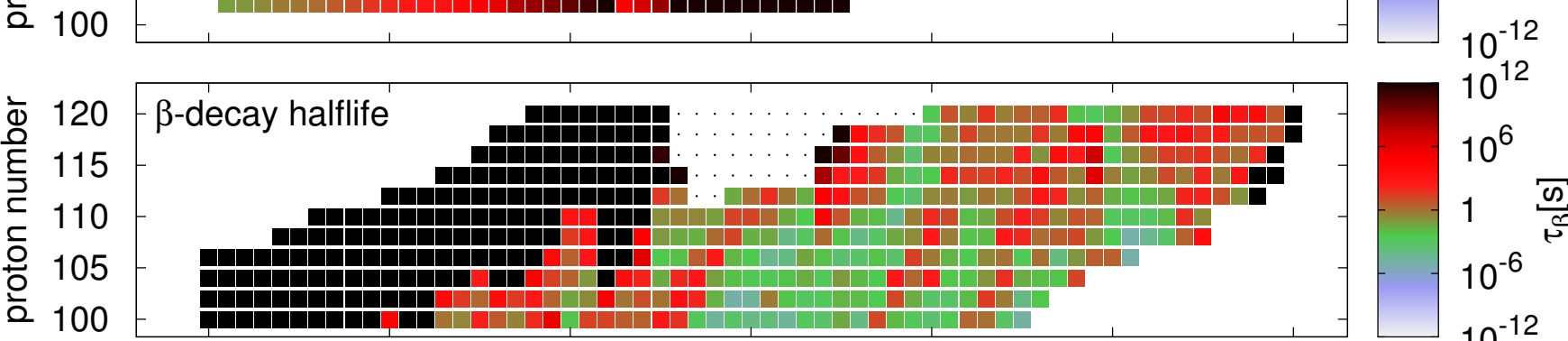

$10^{-12}$

$10^{12}$

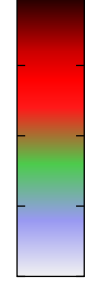

$10^{6}$

$1 \frac{\omega}{n}$ $10^{-6}$

$10^{-12}$

$\begin{array}{ll}\text { ๖े } & 120 \\ \text { 은 } & 115 \\ \text { ㄷ } & 110 \\ \text { 흐 } & 105 \\ \text { 은 } & 100\end{array}$

neutron separation $\mathrm{S}_{\mathrm{H}}$

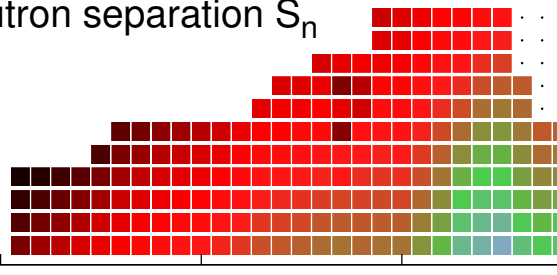

140

160

180

200

neutron number

Fig. 4. Systematics of fission barrier (upper), lifetimes for three different decay channels, and neutron separation energies (lower) over the landscape of SHE computed with SV-min [28. All cases use comparable color (grayscale) scheme. More stability is indicated by red (dark) and less instability by blue color (light gray). The region of triaxial fission, indicated by dots, has not been computed and is thus not considered here.

this parameter which eventually yields an impression of its impact on this observable.

An example of this strategy is shown in figure 6 for the fission barrier $B_{\mathrm{f}}$ of ${ }^{266} \mathrm{Hs}$ as function of the three SHF parameters, pairing strength, effective mass $m^{*} / m$, and symmetry energy $J$ which all were found to have visible effect on $B_{\mathrm{f}}$. Each one of these three SHF parameters has an uncertainty according to the rules of statistical analysis (see section 2.3) which is indicated by a shaded area in the plot. Each parameter produces significant changes of $B_{\mathrm{f}}$. It is interesting to note that all three trends are very close to linear which confirms the expansion about the optional parametrization usually employed in statistical analysis. The amount of variation of $B_{f}$ within the uncer- 


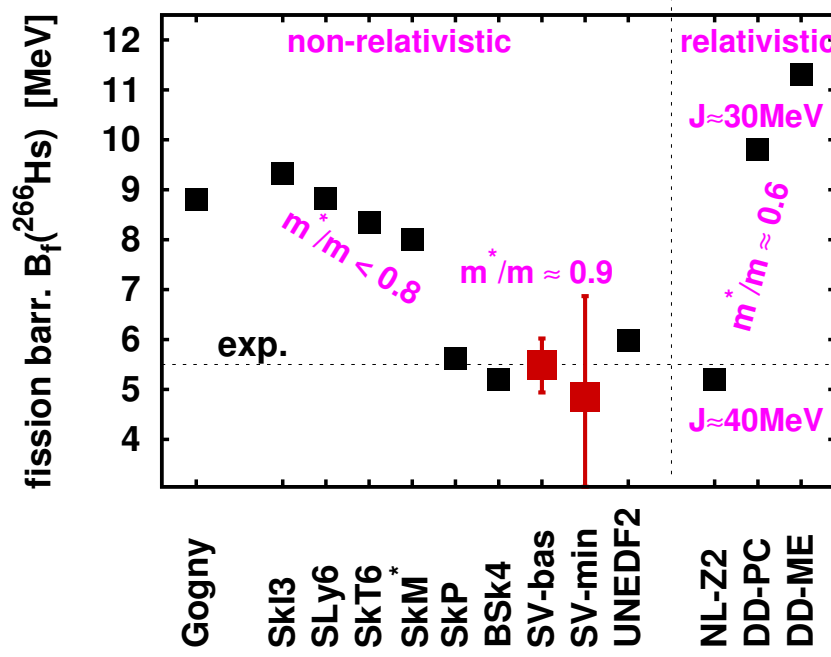

Fig. 5. Fission barrier $B_{\mathrm{f}}$ of ${ }^{266} \mathrm{Hs}(\mathrm{Z}=108)$ for a variety of mean-field models and parametrizations. The values of two NMP (symmetry energy $J$ and effective mass $m^{*} / m$ ) are indicated.

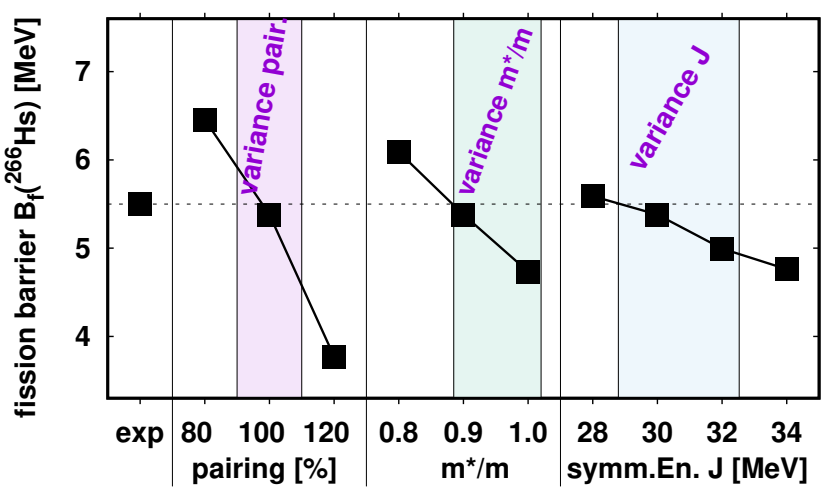

Fig. 6. Fission barrier $B_{\mathrm{f}}$ of ${ }^{266} \mathrm{Hs}(\mathrm{Z}=108)$ for three sets of SHF parametrizations with systematically varied properties, according to 28. The shaded areas indicate the uncertainty of each parameter.

tainty band indicates the strength of the correlation of $B_{f}$ with that parameter and its contribution to its total uncertainty. All three parameters shown in the figure have about comparable and large impact. The effect of pairing strength is plausible because pairing tends to wash out the shell fluctuations and the fission barrier is produced by the fluctuations of shell-correction energy with changing deformation. Effective mass, again, is a quantity strongly related to shell structure and influences $B_{\mathrm{f}}$ via shell-correction energy. The symmetry energy $J$ is a true bulk property with ignorable effect on shell structure. Its influence on $B_{f}$ is mediated through its impact on neutron skin 62,63. which, in turn, plays a role in neck formation.

\subsection{Correlation analysis}

Trend analysis as exemplified in figure 6 is instructive. But it grows cumbersome if more influences are to be com-

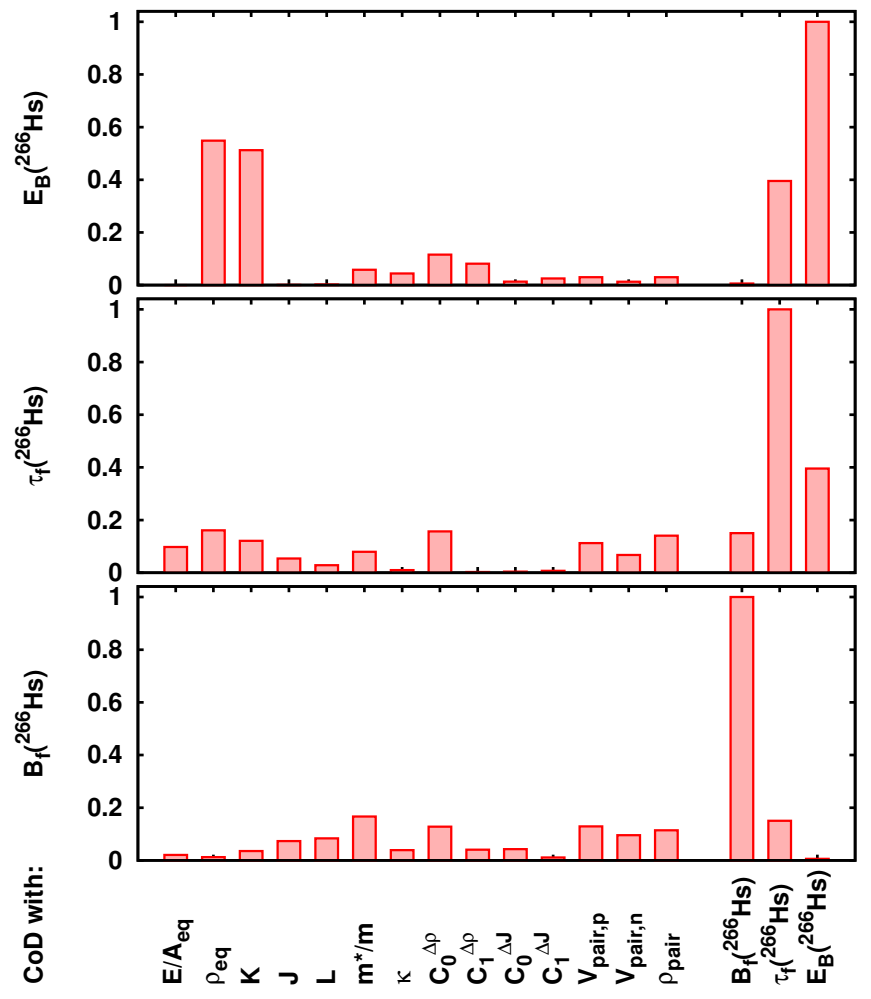

Fig. 7. Correlation of binding energy $E_{B}$, fission barrier $B_{\mathrm{f}}$, and fission halflife $\tau_{\mathrm{f}}$ of ${ }^{266} \mathrm{Hs}$ with the 14 model parameters. The volume parameters are expressed in terms of NMP. Correlations are quantified in terms of CoD.

pared. Correlation analysis in terms of the alignment $r_{A B}$ or the $\mathrm{CoD} r_{A B}^{2}$, see section 2.3. provides a compact measure which allows to overview many ingredients at once. Figure 7 shows the CoD between the SHF parameters and three observables in ${ }^{266} \mathrm{Hs}$, binding energy $E_{B}$, fission lifetime $\tau_{\mathrm{f}}$, and fission barrier $B_{\mathrm{f}}$. All three observables lack a prominent, strong correlation. They rather spread their influences over many parameters. This holds, in particular, for the two fission observables $B_{\mathrm{f}}$ and $\tau_{\mathrm{f}}$. For a proper interpretation, one has to keep in mind that not all SHF parameters are statistically independent from each other and that they have different degrees of determination. The isoscalar, static bulk parameters $E / A_{\text {eq }}, \rho_{\text {eq }}, K$ and the isoscalar surface parameter $C_{0}^{\Delta \rho}$ are tightly fixed by the ground state data. They have too little variance to be used as lever for changing fission properties. Among the parameters which leave more freedom, one has to take care of strong correlations (more than 90\% correlated): between the isovector properties $J$ and $L$ and between the three pairing parameters. With these precautions, we see three somewhat more relevant influencers within the many SHF parameters, namely $J, m^{*} / m$, and pairing, exactly those three whose explicit variation was shown in figure 6 .

The lack of prominent correlations for fission observables is surely a message. But one would still like to learn more about the impact of the model. This can be achieved by considering multiple correlation coefficients (MCC). For example, $V_{\text {pair,p }}, V_{\text {pair,n }}$, and $\rho_{\text {pair }}$ together embrace a 


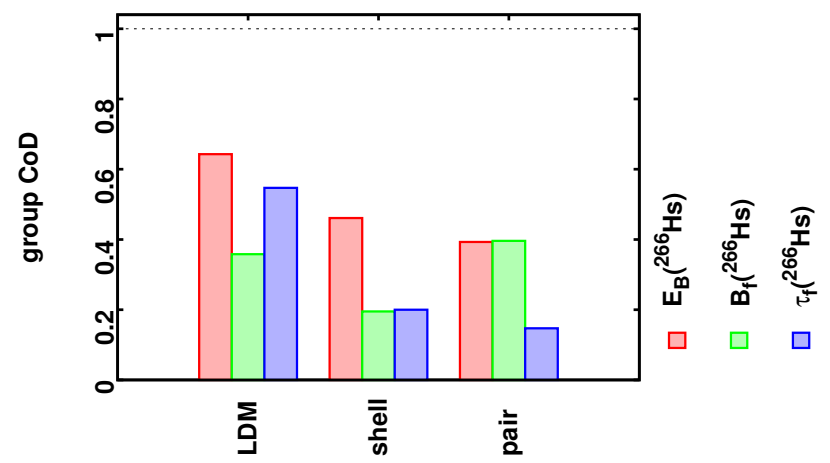

Fig. 8. Multiple correlation coefficient (MCC) of binding energy, fission barrier, as well as fission halflife of ${ }^{266} \mathrm{Hs}$ with groups of model parameters, coined "group CoD". The groups are: $\mathrm{LDM}=E / A_{\mathrm{eq}}, \rho_{\mathrm{eq}}, K, J, L, C_{0}^{\nabla \rho}$, and $C_{1}^{\nabla \rho} ;$ shell $=m / m$, $\kappa_{\mathrm{TRK}}, C_{0}^{\nabla J}, C_{1}^{\nabla J}$; pair $=V_{\text {pair,p }}, V_{\text {pair,n }}, \rho_{\text {pair }}$.

group of parameters related to pairing. One then can ask what is the impact of pairing as a whole (and not only of one single pairing parameter) on fission. This is quantified by the MCC $R_{\mathbf{G} A}^{2}$ of an observable $A$ with groups of parameters $\mathbf{G}=\left(p_{i_{1}}, . ., p_{i_{G}}\right)$ 64]. Values of $R^{2}$, again, range from 0 to 1 , where 0 implies, that those quantities are completely uncorrelated, 1 denotes that the group $\mathbf{G}$ determines the observable $A$ completely. An $R^{2}$ of, say, 0.30 means that $30 \%$ of the variance in $A$ is predictable from G. For a group containing all model parameters, an observable is completely determined, hence $R^{2}=1$. Figure 8 shows MCC of fission properties in ${ }^{266} \mathrm{Hs}$ with three groups of parameters as indicated. The liquid-drop-model (LDM) embraces the static NMP together with isoscalar and isovector surface parameters. It represents the general trends of nuclear bulk properties averaging through shell fluctuations 65]. The "shell" group collects the four parameters having direct impact on level structure, particularly level density and the "pair" group is obviously related to the three pairing parameters in the model. Although the MCC are considerably larger than the single $\mathrm{CoD}$ in figure 7, the basic feature remains, namely that the influences are distributed, here over the three groups under consideration. This illustrates once again that properties of SHE emerge from a subtle interplay of all ingredients of the model. A safe prediction requires careful modeling and counter checks in the SHE region.

\subsection{Trends with nucleon number}

Having explored the impact of model parameters on fission properties, we have finally a quick glance at the system dependence and do this for the fission lifetime $\tau_{f}$. Figure 9 shows the isotopic trends of lifetimes for two SHE, the transactinides No and Hs for which experimental data exist. The data are compared with results from four SHF parametrizations out of the selection of parametrizations in figure 5 where two of them (SV-min and SV-bas) delivered barriers close to data and the other two had

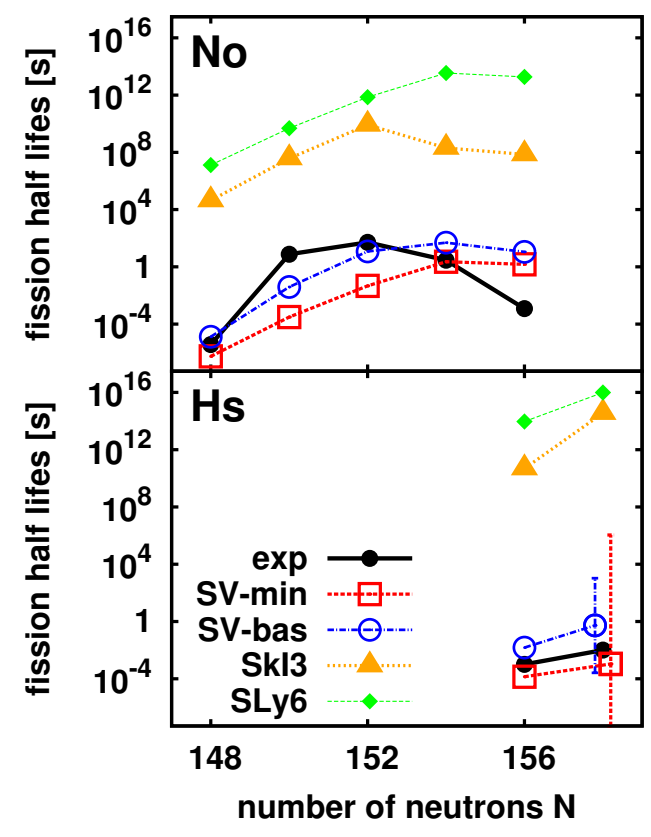

Fig. 9. Fission halflifes $\tau_{f}$ along isotopic chains of two transactinides, No and Hs. Results from four different SHF parametrizations are compared with experimental data (for complete references to experimental fission data see [23]). Approximate extrapolation errors are indicated for ${ }^{266} \mathrm{Hs}$.

too high barriers. The consequence on lifetimes is dramatic as we see in the figure 9 here. SLy6 and SkI3 which overestimate barriers in ${ }^{266} \mathrm{Hs}$ by $3-4 \mathrm{MeV}$ produce lifetimes more than 12 orders of magnitude too large. SVmin and SV-bas whose barriers reach data within less than $1 \mathrm{MeV}$ reproduce lifetimes within 2 orders of magnitude. An estimate of the extrapolation uncertainty is shown for ${ }^{266} \mathrm{Hs}$. It amounts to hefty 9 orders of magnitude for the unrestricted fit SV-min. Only with additional information on response properties built into SV-bas, we come down to about 3-4 orders of magnitude uncertainty. This is a humble figure of what theory can promise at its best. The example thus demonstrates once more the extreme sensitivity of fission lifetimes. A reproduction of data within four orders of magnitude can be considered as success. This goal can be reached with careful calibration of the mean-field model. With the latest generation of SHF parametrizations we dispose now of a reliable description of fission properties of SHE. This can then be used for large-scale surveys in the whole landscape of SHE establishing systematics in the available region and exploring also extremes as, e.g., SHE in the $r$-process region as has been done, e.g., in [23, 66].

\section{Conclusions and outlook}

We have reviewed the theoretical prediction of fission properties of super-heavy elements (SHE) by self-consistent mean-field models. For the latter, we considered particularly the widely used Skyrme-Hartree-Fock (SHF) model. 
The theoretical tools, namely the energy-density functional of SHF, its calibration by least-squares fits with subsequent statistical analysis, and the computation of fission lifetimes, are briefly explained. Already at the formal level, one sees that fission calculations are very involved and the final result emerges from a subtle interplay of several ingredients. Statistical analysis is the tool of choice to disentangle the impact of the various effects and to get an idea of the predictive value of mean-field models.

We have given a summary overview of fission properties in the landscape of SHE in comparison to competing decay channels ( $\alpha$ - and $\beta$-decay, neutron emission). Using a carefully chosen SHF parametrization, the known properties of SHE are well reproduced which gives some confidence in the extrapolation deep into the $r$-process region. Most of the discussion was concerned with analyzing the reliability of SHF for computing properties of SHE. Taking published SHF parametrizations collected over decades of development, yields a disquietingly large spread of predictions. But it shows also that there are many parametrizations which perform very well and this can be related to response properties as, e.g., effective mass or symmetry energy. The impact of these and other properties is worked out by trend and correlation analysis. It is found, quite as expected, that the sensitivity of fission properties is spread over a great multitude of model parameters without clear preference. More can be said when grouping the model parameters into sensible blocks. We have defined three such blocks, a group of liquid-drop model (LDM) properties (static bulk properties without shell effects), a pairing group, and a group determining shell fluctuations. It turns out that the LDM group has largest impact, but not an exclusive one. With comparable weight contributes the pairing group to fission lifetimes and the group of shell effects to the barrier. Finally, we have checked possible trends with system size and charge within the landscape of transactinides. There seems to be no trend in agreement with data. Well fitting parametrizations do that all over this landscape and those who fail fail throughout. Still all results together show that predictions of fission properties have to be taken with care. When extending them to new regions, counter checking with one or two reference points is highly recommended.

\section{Acknowledgments}

First of all, my thank goes to Walter Greiner who inspired and accompanied the studies on self-consistent mean-field models in connection with super-heavy elements. There were many contributors to this research over the years which we cannot list here in detail. But at least two amongst them deserve mentioning, namely J.A. Maruhn and W. Nazarewicz. Their active involvement together with their groups was the necessary condition to work out the large body of work on SHE and nuclear energy density functionals.

\section{References}

1. E.K. Hyde, G.T. Seaborg, in Handbuch der Physik, Vol. XLII (Springer, Berlin, 1957), p. 205

2. J.R. Nix, Annual Review of Nuclear Science 22, 66 (1972)

3. S. Hofmann, G. Münzenberg, Rev. Mod. Phys. 72, 733 (2000)

4. S. Hofmann, F. Heßberger, D. Ackermann, S. Antalic, P. Cagarda, S. wiok, B. Kindler, J. Kojouharova, B. Lommel, R. Mann et al., Eur. Phys. J. A 10, 5 (2001), ISSN 1434-6001

5. Y.T. Oganessian, V.K. Utyonkov, Y.V. Lobanov, F.S. Abdullin, A.N. Polyakov, I.V. Shirokovsky, Y.S. Tsyganov, G.G. Gulbekian, S.L. Bogomolov, B.N. Gikal et al., Phys. Rev. C 70(6), 064609 (2004)

6. Y.T. Oganessian, V.K. Utyonkov, Y.V. Lobanov, F.S. Abdullin, A.N. Polyakov, R.N. Sagaidak, I.V. Shirokovsky, Y.S. Tsyganov, A.A. Voinov, G.G. Gulbekian et al., Phys. Rev. C 74(4), 044602 (2006)

7. K.E. Gregorich, J.M. Gates, C.E. Düllmann, R. Sudowe, S.L. Nelson, M.A. Garcia, I. Dragojević, C.M. Folden III, S.H. Neumann, D.C. Hoffman et al., Phys. Rev. C 74(4), 044611 (2006)

8. J. Dvorak, W. Brüchle, M. Chelnokov, C.E. Düllmann, Z. Dvorakova, K. Eberhardt, E. Jäger, R. Krücken, A. Kuznetsov, Y. Nagame et al., Phys. Rev. Lett. 100(13), 132503 (2008)

9. G. Martínez-Pinedo, D. Mocelj, N. Zinner, A. Keli, K. Langanke, I. Panov, B. Pfeiffer, T. Rauscher, K.H. Schmidt, F.K. Thielemann, Prog. Part. Nucl. Phys. 59(1), 199 (2007)

10. M. Arnould, S. Goriely, K. Takahashi, Physics Reports 450(4-6), 97 (2007)

11. J. Stone, P.G. Reinhard, Prog. Part. Nucl. Phys. 58, 587 (2007)

12. U. Mosel, W. Greiner, Z. Phys. 217, 256 (1968)

13. U. Mosel, W. Greiner, Z. Phys. 222, 261 (1969)

14. P. Ring, Prog. Part. Nucl. Phys. 37, 193 (1996)

15. M. Bender, P.H. Heenen, P.G. Reinhard, Rev. Mod. Phys. 75, 121 (2003)

16. D. Vretenar, A. Afanasjev, G. Lalazissis, P. Ring, Phys. Rep. 409, 101 (2005)

17. J. Erler, P. Klüpfel, P.G. Reinhard, J. Phys. G 38, 033101 (2011)

18. M. Brack, J. Damgård, A.S. Jensen, H.C. Pauli, V.M. Strutinsky, C.Y. Wong, Rev. Mod. Phys. 44, 320 (1972)

19. J.F. Berger, L. Bitaud, J. Decharg, M. Girod, K. Dietrich, Nuclear Physics A 685(1-4), 1 (2001)

20. M. Warda, J. Egido, L. Robledo, Phys. Scr. T 125, 226 (2006)

21. A. Staszczak, A. Baran, J. Dobaczewski, .W. Nazarewicz, Phys. Rev. C 80(1), 014309 (2009)

22. N. Schindzielorz, J. Erler, P. Klüpfel, P.G. Reinhard, G. Hager, International Journal of Modern Physics E 18(4), 773 (2009)

23. J. Erler, K. Langanke, H.P. Loens, G. Martinez-Pinedo, P.G. Reinhard, Phys. Rev. C 85, 025802 (2012)

24. A. Baran, M. Kowal, P.G. Reinhard, L. Robledo, A. Staszczak, M. Warda, Nucl. Phys. A 944, 442 (2015)

25. T. Lesinski, M. Bender, K. Bennaceur, T. Duguet, J. Meyer, Phys. Rev. C 76, 014312 (2007)

26. P.G. Reinhard, Nucl. Phys. A 252, 120 (1975) 
27. P. Klüpfel, J. Erler, P.G. Reinhard, J.A. Maruhn, Eur. Phys. J A 37, 343 (2008)

28. P. Klüpfel, P.G. Reinhard, T.J. Bürvenich, J.A. Maruhn, Phys.Rev. C 79, 034310 (2009)

29. P. Ring, P. Schuck, The Nuclear Many-Body Problem (Springer-Verl., New York, Heidelberg, Berlin, 1980)

30. P. Bonche, H. Flocard, P.H. Heenen, S.J. Krieger, M.S. Weiss, Nucl. Phys. A 443, 39 (1985)

31. S.J. Krieger, P. Bonche, H. Flocard, P. Quentin, M.S. Weiss, Nucl. Phys. A 517, 275 (1990)

32. M. Bender, K. Rutz, P.G. Reinhard, J.A. Maruhn, Eur. Phys. J. A 8, 59 (2000)

33. P.R. Bevington, D.K. Robinson, Data Reduction and Error Analysis for the Physical Sciences (McGraw-Hill, 2003)

34. J. Friedrich, P.G. Reinhard, Phys. Rev. C 33, 335 (1986)

35. T. Niksic, D. Vretenar, G. Lalazissis, P. Ring, Phys. Rev. C 69, 047301 (2004)

36. T. Niksic, D. Vretenar, P. Ring, Phys. Rev. C 78, 034318 (2008)

37. M. Kortelainen, J. McDonnell, W. Nazarewicz, P.G. Reinhard, J. Sarich, N. Schunck, M.V. Stoitsov, S.M. Wild, Phys. Rev. C 85, 024304 (2012)

38. B.A. Brown, Phys. Rev. C 58, 220 (1998)

39. M. Kortelainen, J. McDonnell, W. Nazarewicz, E. Olsen, P.G. Reinhard, J. Sarich, N. Schunck, S.M. Wild, D. Davesne, J. Erler et al., Phys. Rev. C 89, 054314 (2014)

40. G. Hagen, T. Papenbrock, D.J. Dean, M. Hjorth-Jensen, Phys. Rev. Lett. 101, 092502 (2008)

41. P. Navrátil, S. Quaglioni, I. Stetcu, B.R. Barrett, J. Phys. G 36, 083101 (2009)

42. R.F.G. Ruiz, M.L. Bissell, K. Blaum, A. Ekstrm, N. Frmmgen, G. Hagen, M. Hammen, K. Hebeler, J.D. Holt, G.R. Jansen et al., Nature Physics 12, 594 (2016)

43. M. Beiner, H. Flocard, Nguyen Van Giai, P. Quentin, Nucl. Phys. A 238, 29 (1975)

44. J. Bartel, P. Quentin, M. Brack, C. Guet, H.B. Håkansson, Nucl. Phys. A 386, 79 (1982)

45. J. Dobaczewski, H. Flocard, J. Treiner, Nucl. Phys. A 422, $103(1984)$

46. F. Tondeur, M. Brack, M. Farine, J.M. Pearson, Nucl. Phys. A420, 297 (1984)

47. P.G. Reinhard, H. Flocard, Nucl. Phys. A 584, 467 (1995)

48. E. Chabanat, P. Bonche, P. Haensel, J. Meyer, R. Schaeffer, Nucl. Phys. A 635, 231 (1998), Nucl. Phys. A643, 441(E)

49. S. Goriely, M. Samyn, M. Bender, J.M. Pearson, Phys. Rev. C 68, 054325 (2003)

50. S. Goriely, N. Chamel, J.M. Pearson, Phys. Rev. C 88, 061302(R) (2013)

51. J. Dobaczewski, W. Nazarewicz, P.G. Reinhard, Nucl. Phys. A693, 361 (2001)

52. S.A. Glantz, B.K. Slinker, T.B. Neilands, Primer of Applied Regression \& Analysis of Variance (McGraw Hill, 1990)

53. K. Rutz, J.A. Maruhn, P.G. Reinhard, W. Greiner, Nucl. Phys. A590, 680 (1995)

54. R.Y. Cusson, P.G. Reinhard, M.R. Strayer, J.A. Maruhn, W. Greiner, Z. Phys. A 320, 475 (1985)

55. P.G. Reinhard, K. Goeke, Rep. Prog. Phys. 50, 1 (1987)

56. J. Dobaczewski, M. Stoitsov, W. Nazarewicz, P.G. Reinhard, Phys. Rev. C 76, 054315 (2007)

57. P.G. Reinhard, Nucl. Phys. A 306, 19 (1978)
58. P.G. Reinhard, F. Grümmer, K. Goeke, Z. Phys. A317, 339 (1984)

59. V. Viola, G. Seaborg, Journal of Inorganic and Nuclear Chemistry 28, 741 (1966)

60. K.J. Pototzky, J. Erler, P.G. Reinhard, V.O. Nesterenko, subm. Eur. Phys. A (2010)

61. A.S. Umar, V.E. Oberacker, C.J. Horowitz, P.G. Reinhard, J.A. Maruhn, Phys. Rev. C 92, 025808 (2015)

62. P.G. Reinhard, W. Nazarewicz, Phys. Rev. C 81(5), $051303(2010)$

63. W. Nazarewicz, P.G. Reinhard, W. Satuła, D. Vretenar, Eur. Phys. J. A 50, 20 (2014)

64. P.D. Allison, Multiple Regression: A Primer (Sage Publications, 1998)

65. W.D. Myers, W.J. Swiatecki, Annual Review of Nuclear and Particle Science 32, 309 (1982)

66. I. Petermann, K. Langanke, G. Martinez-Pinedo, P.G.R. I.V. Panov, F.K. Thielemann, Eur. Phys. J. A 48, 122 (2012) 